Vol. 30, Issue 12, December 2007

Editorials

602 Cetuximab and Chemotherapy for Patients with Unresectable CRC Liver Metastasis: Are We Changing the Natural Course of the Disease? Ramos, F.J.; Tabernero, J. (Barcelona)

606 Five Years of Clinical Experience with Metronomic Chemotherapy: Achievements and Perspectives Emmenegger, U.; Kerbel, R.S. (Toronto, ON)

Original Articles

611 Ovarian Cancer Treatment Reality in Northern Rheinland-Pfalz (Germany). Suboptimal Surgical Treatment as a Possible Cause for Inferior Survival Weide, R.; Arndt, M.; Pandorf, A.; Heymanns, J.; Thomalla, J.; Köppler, H. (Koblenz)

621 Cost-Benefit Assessment of Antifungal Prophylaxis with Liposomal Amphotericin B in Neutropenic Patients

Penack, O.; Reinhold, T.; Thiel, E.; Blau, I.W. (Berlin)

629 Metronomic Anti-Angiogenic Therapy with Capecitabine and Celecoxib (CC) in Advanced Tumor Patients - Results of a Phase II Study Steinbild, S.; Arends, J.; Medinger, M.; Häring, B.; Frost, A.; Drevs, J.; Unger, C.; Strecker, R.; Hennig, J.; Mross, K. (Freiburg i.Br.)

637 Cetuximab in Combination with 5-Fluorouracil, Leucovorin and Irinotecan as a Neoadjuvant Chemotherapy in Patients with Initially Unresectable Colorectal Liver Metastases Min, B.S.; Kim, N.K.; Ahn, J.B.; Roh, J.K.; Kim, K.S.; Choi, J.S.; Cha, S.H.; Kim, H. (Seoul)

\section{Clinical Cases}

645 The Diagnosis of C-Kit Negative GIST by PDGFRA Staining: Clinical, Pathological, and Nuclear Medicine Perspective Sevinc, A.; Camci, C.; Yilmaz, M.; Buyukhatipoglu, H. (Gaziantep)
Band 30, Heft 12, Dezember 2007

Editorials

602 Cetuximab und Chemotherapie für Patienten mit nicht resektablen CRC-Lebermetastasen: Ändern wir den Krankheitsverlauf?

Ramos, F.J.; Tabernero, J. (Barcelona)

606 Fünf Jahre klinische Erfahrungen mit metronomischer Chemotherapie: Erreichtes und Perspektiven Emmenegger, U.; Kerbel, R.S. (Toronto, ON)

Originalarbeiten

611 Behandlungsrealität von Patientinnen mit Ovarialkarzinom im nördlichen Rheinland-Pfalz. Suboptimle chirurgische Behandlung als mögliche Ursache einer niedrigeren Überlebensrate Weide, R.; Arndt, M.; Pandorf, A.; Heymanns, J.; Thomalla, J.; Köppler, H. (Koblenz)

621 Kosten-Nutzen-Analyse der Prophylaxe von Invasiven Mykosen mit Liposomalem Amphotericin B bei Neutropenen Patienten Penack, O.; Reinhold, T.; Thiel, E.; Blau, I.W. (Berlin)

629 Metronomische, antiangiogene Therapie mit Capecitabin und Celecoxib (CC) bei Patienten mit fortgeschrittenen Tumorerkrankungen - Ergebnisse einer Phase II Studie Steinbild, S.; Arends, J.; Medinger, M.; Häring, B.; Frost, A.; Drevs, J.; Unger, C.; Strecker, R.; Hennig, J.; Mross, K. (Freiburg i.Br.)

637 Cetuximab in Kombination mit 5-Fluorouracil, Leucovorin und Irinotecan als neoadjuvante Chemotherapie bei Patienten mit primär nicht resektablen kolorektalen Lebermetastasen Min, B.S.; Kim, N.K.; Ahn, J.B.; Roh, J.K.; Kim, K.S.; Choi, J.S.; Cha, S.H.; Kim, H. (Seoul)

Kasuistiken

645 Diagnose C-Kit-negativer GIST durch PDGFRAFärbung: klinische, pathologische und nuklearmedizinische Aspekte Sevinc, A.; Camci, C.; Yilmaz, M.; Buyukhatipoglu, H. (Gaziantep) 


\section{Vol. 30, Issue 12, December 2007}

651 Severe Hyponatremia in a Patient with Mantle Cell Lymphoma treated with Bortezomib: A Case Report and Review of the Literature

Brodmann, S.; Gyr Klaas, E.; Cathomas, R. (Chur);

Girardi, V. (Winterthur); von Moos, R. (Chur)

Review Article

657 Gemtuzumab Ozogamicin (Mylotarg) for the Treatment of Acute Myeloid Leukemia - Ongoing Trials

Gleissner, B. (Homburg); Schlenk, R.(Ulm); Bornhäuser, M. (Dresden); Berdel, W.E. (Münster)

Erratum

662 Retraction: Intraoperative Touch Imprint Cytological Analysis of Sentinel Lymph Nodes for the Presence of Metastases in Breast Cancer

\section{Acknowledgement to Reviewers}

664 PharmaForum

672 PharmaNews / PharmaTicker

643 Imprint

675 Meetings and Conferences

678 Author Index Vol. 30, 2007

681 Subject Index Vol. 30, 2007

III Contents Vol. 30, 2007 (following page 684)

Forthcoming papers are listed on page 677.

\section{Band 30, Heft 12, Dezember 2007}

651 Schwere Hyponaträmie bei einer mit Bortezomib behandelten Patientin mit Mantelzell-Lymphom: Ein Fallbericht mit Literaturübersicht Brodmann, S.; Gyr Klaas, E.; Cathomas, R. (Chur); Girardi, V. (Winterthur); von Moos, R. (Chur)

Übersichtsarbeit

657 Gemtuzumab Ozogamicin (Myelotarg) zur Behandlung der akuten myeloischen Leukämie - laufende Studien Gleissner, B. (Homburg); Schlenk, R.(Ulm); Bornhäuser, M. (Dresden); Berdel, W.E. (Münster)

Erratum

662 Widerruf: Intraoperative Schnelldiagnostik von Sentinel-Lymphknoten zur Darstellung von Brustkrebsmetastasen

663 Dank an die Gutachter

664 PharmaForum

672 PharmaNews / PharmaTicker

643 Impressum

675 Tagungen und Kongresse

678 Autorenverzeichnis Band 30, 2007

683 Sachwortverzeichnis Band 30, 2007

VIII Inhalt Band 30, 2007 (nach Seite 684)

Einen Ausblick auf den Inhalt der kommenden Hefte finden Sie auf Seite 677.

\section{KARGER}

Fax +497614520714

E-mail Information@Karger.de

www.karger.com (c) 2007 S. Karger GmbH, Freiburg

Artikel (Volltext) und Inhaltsverzeichnisse

sowie das vorläufige Inhaltsverzeichnis des nächsten Heftes: www.karger.com/onk_bk.htm 